Portable Instrument for In-vivo Infrared Oxymetry Using Spread-Spectrum Modulation Stefano

Trevisan, Matteo Bavera and Mario E Giardini

Journal of Instrumentation 2 (2007) P04005

This is an author-generated version

The final publication is available at http://iopscience.iop.org/1748-0221/2/04/P04005/

DOI : $10.1088 / 1748-0221 / 2 / 04 / P 04005$ 


\section{Portable Instrument for In-vivo Infrared Oxymetry Using Spread- Spectrum Modulation}

Stefano Trevisan, matteo Bavera and Mario E Giardini

\section{INTRODUCTION}

From an optical point of view, biological tissue can be considered as an absorbing matrix in which a high number of inhomogeneities, which act as light scatterers, are present [1]. When collimated near infrared (NIR) light is injected into the tissue and the collimated transmission is observed, light losses are mostly due to scattering rather than to absorption [2]. The spectral analysis of the backscattered light is, in the medical field, normally referred to as NIRS (Near InfraRed Spectroscopy) [3].

In the NIR region, the optical absorption is dominated by hemoglobin, the blood constituent that carries oxygen by fixing it in an oxygenated form (oxyhemoglobin) in the lungs, and releasing it into the tissue, thus reverting to a deoxygenated form (deoxyhemoglobin); a quantitative determination of the differential absorption spectrum may give information on tissue oxygen content [4]. This is the main goal of NIRS. 
Oxygen metabolism is most certainly a crucial problem in many fields of medical activity. Sport medicine, rehabilitative cardiology, intensive care, anaesthesia, tumor detection, rely on some form of metabolic oxygen analysis. Using NIRS, the key issue of real-time non-invasive evaluation of blood content and oxygenation of muscle in a non-stationary condition, such as upon exercise or during respiratory manoeuvres, can be approached by optical transmission measurements.

The possibility of performing NIRS measurements without hindering other patient activities could open a practical way of accessing oxygen metabolism information related to everyday life activities, to infrequent events, or to hostile environments (such as required by high altitude, high pressure or microgravity medicine). In strongly feeling the need for a portable, compact and yet mechanically robust NIRS acquisition system, we require the instrument to present a number of measurement channels sufficiently high for a complete clinical picture. The large number of channels should be complemented by flexible input stages, in order to accommodate for different NIRS probe designs and for auxiliary non-NIRS signals. The system should be able to operate reliably in a non-controlled and non-conditioned environment. And, finally, it should provide for some sort of remote data transmission, at least for the most time-critical signals, such as heartbeat or breathing rate.

In this paper, a highly compact and portable instrument, dedicated to optical characterization of tissue, will be presented. The instrument has been developed within the IRIS (InfraRed In-vivo Spectrometer) series [5,6] property of the Italian National Institute for Physics of Matter - INFM, and it has been called IRIS-3. By using a nonstandard source multiplexing scheme, the instrument reaches a signal quality compatible with high-end research applications. 


\section{PRINCIPLE OF OPERATION}

NIRS instruments inject NIR light into the tissue using a set of light sources at different wavelengths simultaneously applied to the surface of the tissue, and detect the light intensity backscattered by the tissue on suitable detectors, again applied to the tissue. The backscattered light is subsequently analyzed using specialized wavelengthselective algorithms [7]. However, for such analysis to be medically significant, the measurement geometries imply a very low backscattered light intensity, and to the

present day there are no technologically viable solutions for wavelength-selective detectors with sufficient detectivity and dynamics, and yet mechanically- and costcompatible with the application. NIRS systems therefore associate each backscattering response component to the corresponding light source using non-selective detectors and a suitable modulation-demodulation algorithm. Such approach, when applied to portable equipment, that needs to operate in an electrically and optically unconditioned environment, has up to now implied the need for efficient, and therefore bulky and heavy, shielding of the spectrometer electronics.

In our system, $\mathrm{N}$ light sources (with $\mathrm{N}$ ranging from 2 to 8 ) are modulated using a set of $\mathrm{N}$ functions $f_{n}(\mathrm{n}=1 \ldots \mathrm{N})$ with vanishing cross-correlation. They are simultaneously applied to the tissue, and the signal is detected by a single detector. The detector signal $S$ can be expressed as:

$$
S=\sum_{n=1 \ldots N} a_{n} f_{n}
$$

where $a_{n}$ are the signals associated to the single sources. In order to associate each component to the appropriate source, the signal is multiplied independently by the modulation functions and time-integrated (in practice, low-pass filtered). For the n-th source: 


$$
\begin{aligned}
S_{n} \quad & =\int f_{n} S d t \\
& =a_{n} \int f_{n}^{2} d t
\end{aligned}
$$

as the modulation functions cross correlations vanish, and only the n-th term of the sum is non-null.

When the modulation functions are chosen as sinusoids or square waves with different frequencies, such correlation multiplexing is known as frequency multiplexing by lock-in detection [8]. In such case, if the signals are digitized, in order to obtain an acceptable signal quality and low channel crosstalk, the signal sampling period must be significantly lower and the integration time significantly higher than the sinusoid period. This may easily require, even for low sinusoid frequencies, fast analog-to-digital converters (ADC) and processors with high clock rates. In old implementations of microcontroller-based digital front-ends for NIRS, in order to implement effectively a digital lock-in scheme centered around $1 \mathrm{kHz}$ on a 4 source-detector pair apparatus, we have felt the need to employ video-rate ADC's, allocating a corresponding computing power [9].

A more subtle drawback of digital lock-in for NIRS is related to the fact that, if the modulation frequencies are kept in the $\mathrm{kHz}$ range in order to employ cost-effective processor and ADC technologies, they are quite too near to the frequency of common narrowband noise sources in a medical environment, i.e. ambient light, electrosurgical unit discharges (in the 10-30 kHz range) and high-power switching-mode supplies. For portable equipment, we may add to this the fact that the instrument operates in an environment that cannot always be fully characterized with respect to narrow-spectrum electromagnetic interference in the $\mathrm{kHz}$ range. 
A much more effective choice of the modulation functions $f_{n}$ comes therefore from the consideration that independent noise sources show, by definition, high autocorrelation and low cross-correlation. The $\mathrm{f}_{\mathrm{n}}$ can therefore be chosen as noise-like signals, presenting with respect to sinusoids the advantage of low cross-correlation not only between each other, but also with random and periodic noise sources. In fact, the only signal that, with such choice for $\mathrm{f}_{\mathrm{n}}$, could contaminate the demultiplexing of the $\mathrm{n}$ th source is a noise similar to $f_{n}$ itself, and such a noise is extremely unlikely to occur. Upon multiplication with $f_{n}$ in the demultiplexing process, the spectrum of any other noise source is "smeared out" over the whole frequency range occupied by $f_{n}$, and the integration will reduce the noise amplitude to minimal levels. Such modulation, known as "spread spectrum" [10], is used in telecommunications mainly for data security reasons, as only the knowledge of $f_{n}$ and of its synchronization allows a reconstruction of the signal associated to the $n$-th source.

It would go far beyond the scope of this paper to present even a basic theory of spread spectrum. From a more functional point of view, the attention can be focused on employing as $f_{n}$ functions which oscillate between 0 (source off) and 1 (source on) states in a pseudo-random sequence with minimal cross-correlation. We also require the functions to switch between 0 and 1 on multiples of a common time interval (i.e., we require the 0-1 sequences to change state synchronously with a common clock). We also require the sequences to be periodic with a common period. These two requirements allow us to precalculate such sequences, to store them in a look-up table in a microcontroller, and to use such microcontroller to implement the modulation and demodulation process.

With respect to other solutions found in the literature for portable NIRS, the choice of spread-spectrum modulation functions imply therefore simpler shielding 
lower analog-to-digital conversion rates and consequent efficient use of computing power, thus reducing the overall instrument complexity and weight.

Spread-spectrum modulation functions that follow these requirements are available in the literature. In IRIS-3, we have employed $f_{n}$ developed in 1967 by Magnavox Corporation, known as "gold codes".

\section{ELECTROMECHANICAL INSTRUMENT STRUCTURE}

IRIS-3 is composed by a battery-powered stand-alone portable unit, to which emitter/receiver probes are connected. Two source and two detector amplifier connectors are provided. As in the literature most quantitative algorithms rely on the backscattering data at 4 different wavelengths [7], each source connector can drive up to 4 sources, for a total of 8 independent sources. For signal detection, two preamplified and filtered software-configurable channels are available. Two auxiliary non-amplified analog inputs are also accessible for connection to external auxiliary sensors.

A functional block diagram of the portable unit is represented in Fig. 1. The core of the instrument consists in a single-chip RISC microcontroller (PIC16F877, Microchip Technology Inc., U.S.A.), with embedded 8K FLASH program memory, 368 data registers and mixed-signal peripherals. In particular the processor has been configured in order to activate the on-chip analog-to-digital converters (ADC's), universal synchronous-asynchronous serial transmitter-receiver (USART), serial peripheral interface (SPI), 256-byte EEPROM, timers and interrupt generator.

The unit derives its supply from a rechargeable NiMH battery. The microcontroller monitors and switches the supply on and off to the rest of the unit as needed. It can also enter a low-consumption standby mode, in order to keep the 
instrument under full software control even when in the "off" state. The battery duration depends on the actual measurement protocol and, for a standard NIRS acquisition, it is in excess of 24 hours.

The microcontroller is interfaced to the two source connectors through two fourchannel current boosters, specifically designed for direct interfacing to LED's. The current boosters are switched on and off with the appropriate sequence and the resulting transmitted light is detected and preamplified by two independent detector channels. The amplified signals are then fed to the microcontroller using the embedded ADC's. The data is demultiplexed and filtered digitally, and stored on a 1 MByte static RAM bank. A disposable lithium backup battery provides for permanent data storage when the instrument is in the "off" state.

The measured data can be downloaded to external mass storage using the microcontroller USART, accessible through the I/O and control connector. For highbandwidth or real-time measurements, the instrument can transmit the data on a monodirectional $433 \mathrm{MHz}$ radio link.

The serial connection is also employed to configure the input stages and to set up the measurement parameters using a short program written in a dedicated high-level definition language, that defines signals, timings, filtering and storage. The program is uploaded to the microcontroller on the serial link, stored on the microcontroller in an embedded EEPROM, and executed by a real-time interpreter. An external user interface, running on a personal computer under a 32-bit Microsoft Windows environment implements all the instrument setup, the measurement protocol definition and debugging, the configuration upload and the data download functions. 
A 4-key keyboard, a 4-character alphanumeric LED display on the instrument front panel and a piezo beeper provide the elementary I/O features necessary for standalone operation.

A slow 64 kByte SPI EEPROM bank implements maintenance data logging capabilities.

The instrument is enclosed in a $145 \times 84 \times 35 \mathrm{~mm}$ plastic case with internal conductive shielding, and weighs $370 \mathrm{~g}$ (batteries included).

\section{THE DETECTOR FILTER/AMPLIFIERS}

As the instrument should be suitable for different NIRS measurement protocols, involving different probe designs (that may also include non-NIRS signals), the two detector channels have been equipped with flexible software-configurable amplifiers. A functional block diagram of the detector inputs, as seen by the user, is reported in Fig. 2.

One of the two detector inputs can be configured as a high-sensitivity transconductance amplifier, for direct photodiode input, or as a voltage follower, for preamplified detectors. The input has actually a summing structure, and can add differential signals from a high-impedance differential amplifier, whose input pins are available on the source probe connector. The summing structure of the input allows simultaneous operation of the transimpedance/voltage and of the differential inputs, for background subtraction or for baseline restoration. Outputs are provided for active driving of the probe shield and active feedback and compensation of the common mode signal, as in common practice for the detection of bioelectric signals and for bridge readout [11]. 
The differential amplifier sensitivity is sufficient for the detection of the heartbeat signal in an Einthoven triangle configuration.

An integration-subtraction-mode high-pass filter, with a cutoff frequency below $1 \mathrm{~Hz}$, can be coupled to the input stage for background subtraction [12]. As it works by integration and direct subtraction of the input current, it can compensate continuous backgrounds a few orders of magnitude higher than the actual signal, with practically no offset amplification issues.

The output of the first stage is then fed to a software-controllable variable gain $(0-35 \mathrm{~dB})$ voltage follower, directly followed by a bypassable high-pass filter. The signal is finally sent to the microcontroller for AD conversion.

The other detector input presents a somewhat simplified structure, leaving free pins on the source probe connector, used for two auxiliary non-preamplified analog channels with 0-4V dynamic range.

Low noise power supplies are available on all connectors, and are controlled by the microprocessor.

Both channels can therefore essentially be configured as voltage gain or as transconductance AC or DC coupled inputs, with differential input and active shielding capabilities. The bandwidth is in excess of $1 \mathrm{kHz}$.

\section{FUNCTIONAL TESTS}

As no in-vitro benchmarking test has been univocally established in the relevant NIRS literature to define a formal signal-to-noise ratio, the instrument has been evaluated against an in-vivo reference protocol known as occlusive forearm ischemia 
[13]. Even though requiring a subjective assessment and being therefore somewhat qualitative in providing a figure for system performance, such test is well established in the literature and NIRS laboratory practice as strongly indicative of the significance of the measured data towards clinical applicability.

In such protocol, the patient is at rest, supine, in a stationary state with respect to blood pressure, heart rate and muscle fatigue. A pneumatic tourniquet is applied on the patient arm and is inflated in order to shut off completely the blood supply to the forearm (ischemia). During the ischemia, oxygen consumption progressively deoxygenates the blood in the forearm. The forearm vessels respond to the absence of flow by trying to increase their diameter, hindered into this by the mechanical constraint that the total blood volume in the arm is kept constant by the occlusion. When, after a few minutes, the tourniquet is released, the blood starts flowing again through the forearm. Since all volume constraints are removed, the vessels can dilate freely, and blood volume and oxygenation overshoot the initial value, that is normally recovered a few minutes after ischemia removal.

For this test we have attached to the instrument a NIRS probe consisting in a set of 4 LEDs with light emission peaks at $660 \mathrm{~nm}, 700 \mathrm{~nm}, 850 \mathrm{~nm}$ and $880 \mathrm{~nm}$ mounted on a plastic support at a distance of $15 \mathrm{~mm}$ from a $7 \mathrm{~mm}^{2}$ PIN photodiode (Siemens BPW34). The LEDs and the photodiode have been connected directly to the instrument source and probe connectors respectively, via $1.2 \mathrm{~m}$ shielded cables. The detector input has been configured as a DC-coupled transconductance amplifier. The probe has been fixed on the forearm under test, onto the biceps muscle, using a strip of medical adhesive tape, and shielded from ambient light using a dark cloth cuff.

The results shown in Fig. 3 have been measured on a male 32-year old healthy volunteer. As the interest is focused on signal quality, the figure reports the transmitted 
intensities rather than the calculated oxygenation and perfusion variations, in order not to introduce model-dependent quantities. The intensities, sampled with a $0.5 \mathrm{~s}$ time interval, have been normalized to the respective baseline values.

The measured signals are consistent with the literature. During tourniquet inflation (A) blood is actively pumped into the arm, and the overall transmittance decreases. As the ischemia goes on, the hemoglobin in the blood is progressively converted from the oxidized to the reduced form. The two hemoglobin forms present the same optical absorption at $800 \mathrm{~nm}$; below such wavelength the absorption is strongly dominated by the reduced form, while above the oxidized prevails mildly [14]. As the blood is constrained to a constant volume and deoxygenated, therefore, the tissue transmittance below $800 \mathrm{~nm}$ decreases, while above $800 \mathrm{~nm}$ it remains almost constant, with a very mild increase, and the signals reflect such behaviour. When the ischemia is released (B), after a rapid transmittance decrease due to the vasodilation (reactive hyperemia, C), the baseline values are slowly recovered (D).

We note that, even with such a simple probe, which does not implement any form of photodetector preamplification, the signal is clean. The noise level is sufficiently low as to allow a full recovery of the clinical picture from the transmittance curves.

An even stronger benchmark has been performed by applying the same probe to one of the quadriceps muscles (vastus lateralis) on the volunteer while pedalling on a bicycle. The light backscattered by the muscle at $850 \mathrm{~nm}$ during such exercise is reported in Fig. 4. With the limitations of a qualitative test, the direct visibility of the periodic modulation of such light, synchronous with the pedal cycle and due to the cyclic oxygenation and deoxygenation of the muscle during the exercise, is known to be among the most difficult clinical benchmarks for NIRS systems. 


\section{CONCLUSIONS}

A portable acquisition system, optimized for 8-source 2-detector NIRS has been described in detail. The exploitation of a non-standard digital correlation source multiplexing scheme allows a compact, robust and computationally effective signal detection even with the simple analog hardware and low-cost, low-power processor employed. The system, tested on clinical benchmark protocols, shows a performance adequate for high-end clinical applications.

Aspects of the technology presented in this work have been protected by INFM under PCT patent WO/2003/014714.

\section{REFERENCES}

[1] V. Tuchin, Tissue Optics, SPIE, Bellingham (U.S.A), 2000

[2] F.F. Jöbsis, "Non-invasive, infra-red monitoring of cerebral and myocardial oxygen sufficiency and circulatory parameters”, Science 198, 1264-1267 (1977)

[3] H. Owen-Reece, M. Smith, C.E.Elwell and J.C. Goldstone, "Near-infrared spectroscopy”, Br J Anaesth 82 (3), 418-426 (1999)

[4] S. Wray, M. Cope, D.T. Delpy and J.S. Wyatt, , and E.O.R. Reynolds, "Characterization of the near infrared absorption spectra of cytochrome aa3 and hemoglobin for the non-invasive monitoring of cerebral oxygenation", Biochim Biophys Acta 933, 184-192 (1988) 
[5] M.E.Giardini, M.Corti, G.G.Guizzetti, P.Lago, A.Gelmetti and G.Danese, "microcontroller-based digital front-end for near-infrared spectroscopy", SPIE Proc 3911, 338-344 (2000)

[6] M.E.Giardini, M.Corti, P.Lago and A.Gelmetti, "portable microcontroller-based instrument for near infrared spectroscopy", SPIE Proc 3911, 250-255 (2000)

[7] S.J. Matcher, C.E. Elwell, C.E.Cooper and D.T. Delpy, "Performance comparison of several published tissue near-infrared spectroscopy algorithms", Anal Biochem 227, pp. $54-68(1995)$

[8] M.L. Meade, Lock-in amplifiers: principles and applications, Peter Peregrinus Ltd., London (U.K.), 1983

[9] G.G.Guizzetti, G.Danese, F.Leporati and M.E.Giardini, "An intelligent acquisition system for near infrared spectroscopy", in Medical Diagnostic Techniques and Procedures, M.Singh et al. (eds), pp. 182-187, Narosa Publishing House, New Delhi, India (1999)

[10] R.E. Ziemer, R.L. Peterson, D.E.Borth, Introduction to spread-spectrum communications, Prentice Hall Inc, Upper Saddle River (U.S.A), 1995

[11] "Low level measurements”, Reference manual, Keithley Instruments Inc., U.S.A. 
[12] "Photodiode monitoring with op amps", Application Bulletin AB-075, Burr-Brown Corporation, U.S.A.

[13] N.B. Hampson and C.A.Piantadosi, "Near infrared monitoring of human skeletal muscle oxygenation during forearm ischemia”, J Appl Physiol 64, 2449-2457 (1988)

[14] M. Cope, Ph.D. thesis, University College London, U.K. (1991) 


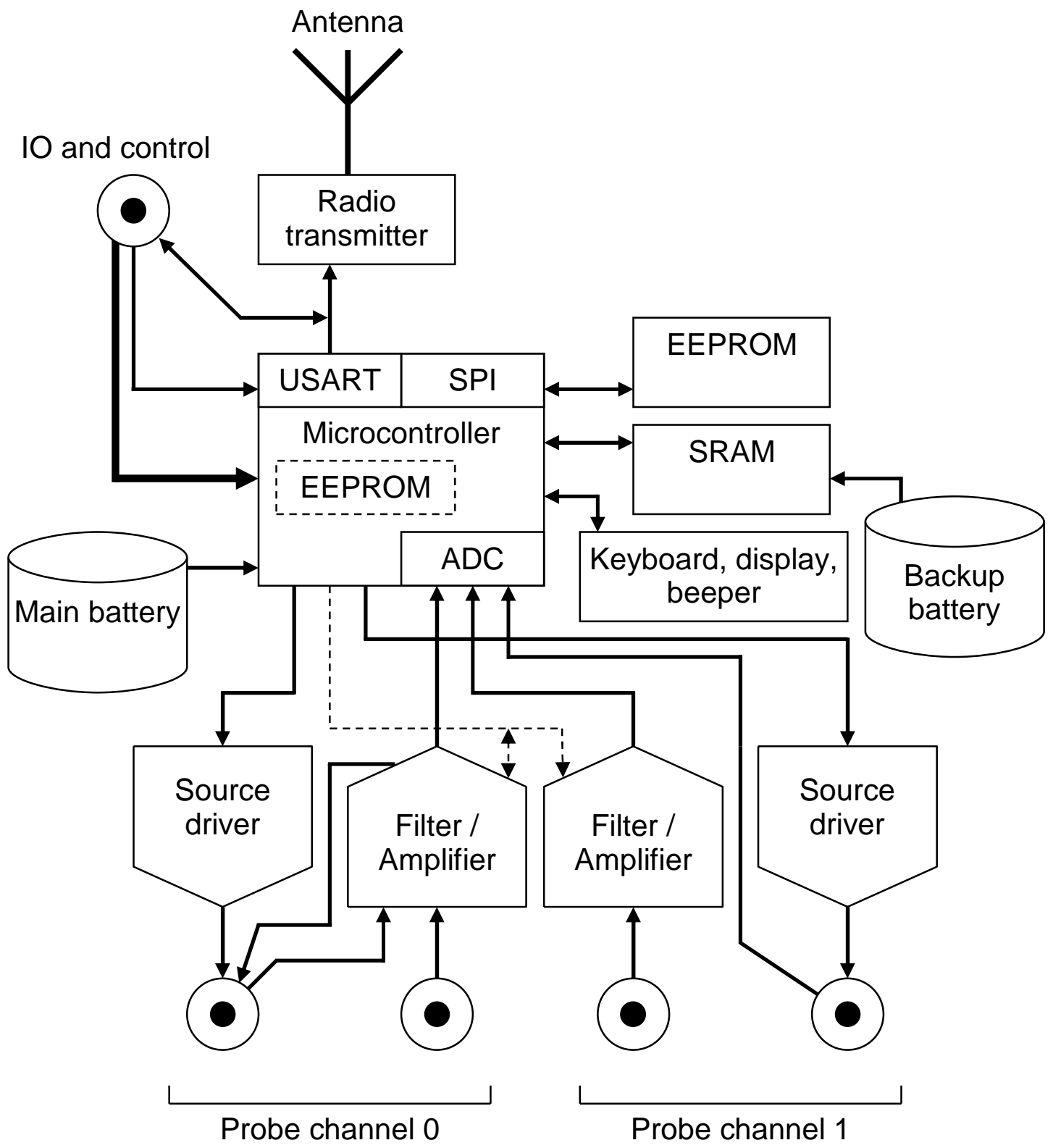



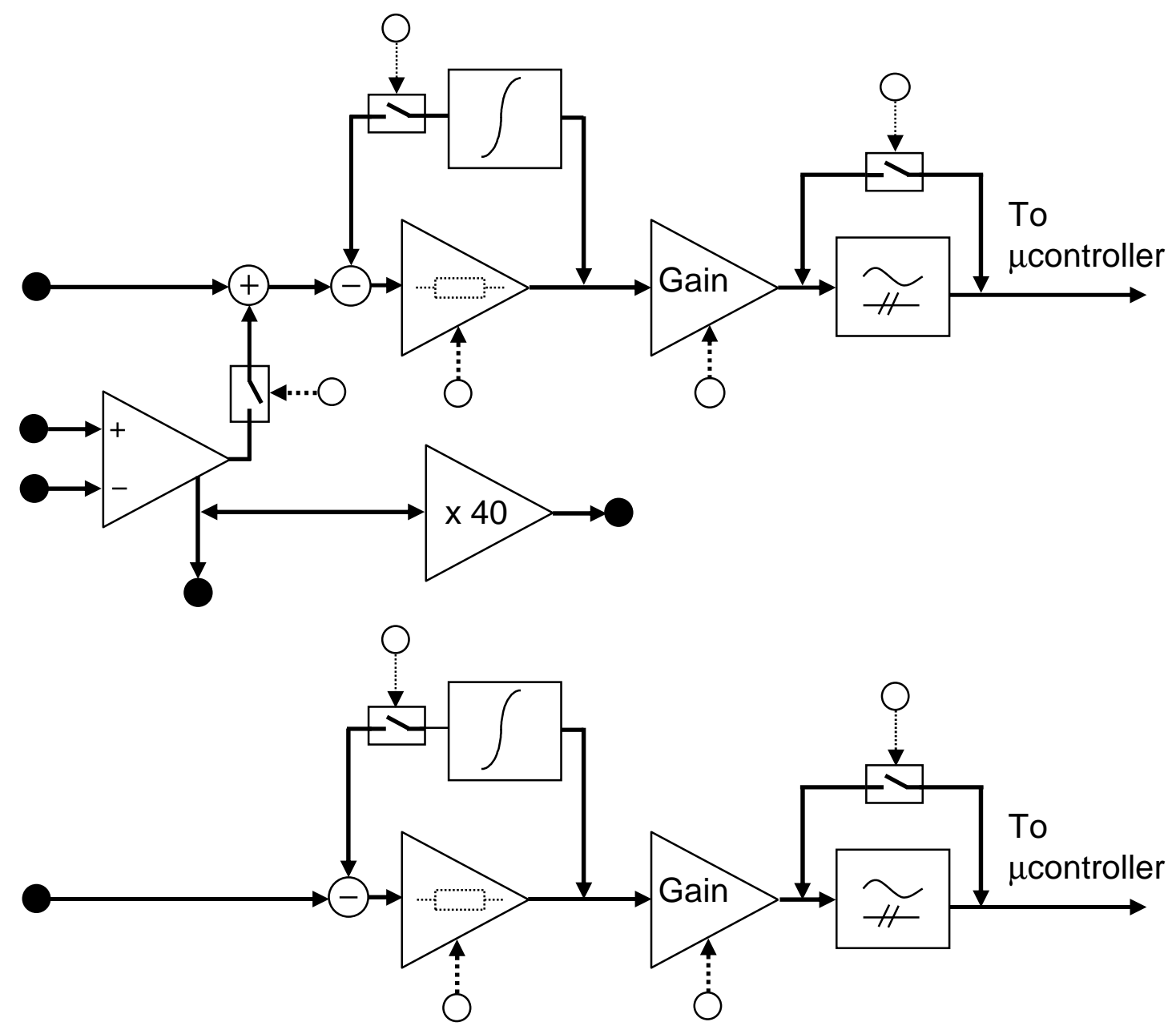


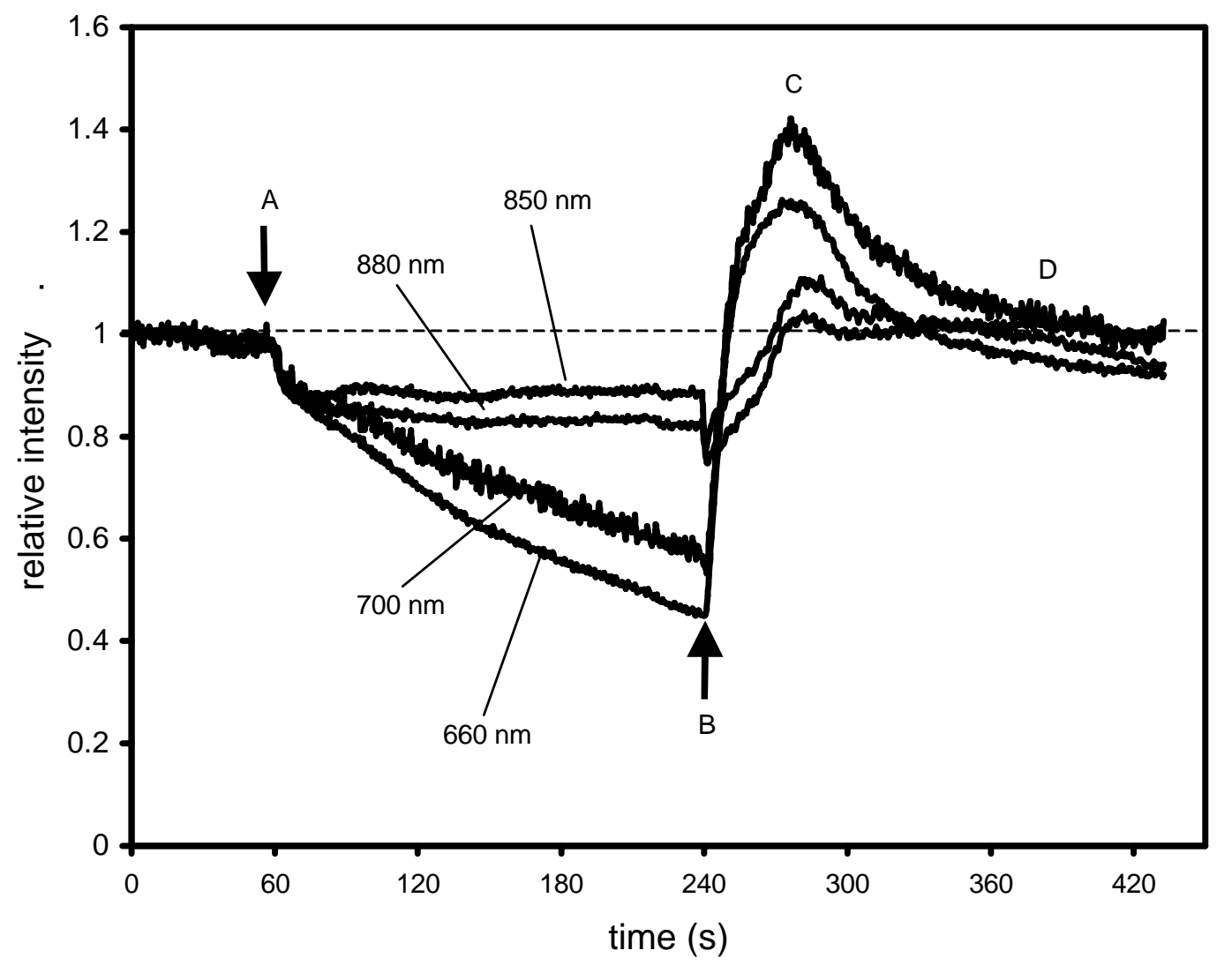


\begin{tabular}{|c|}
\hline 4 \\
UP
\end{tabular}

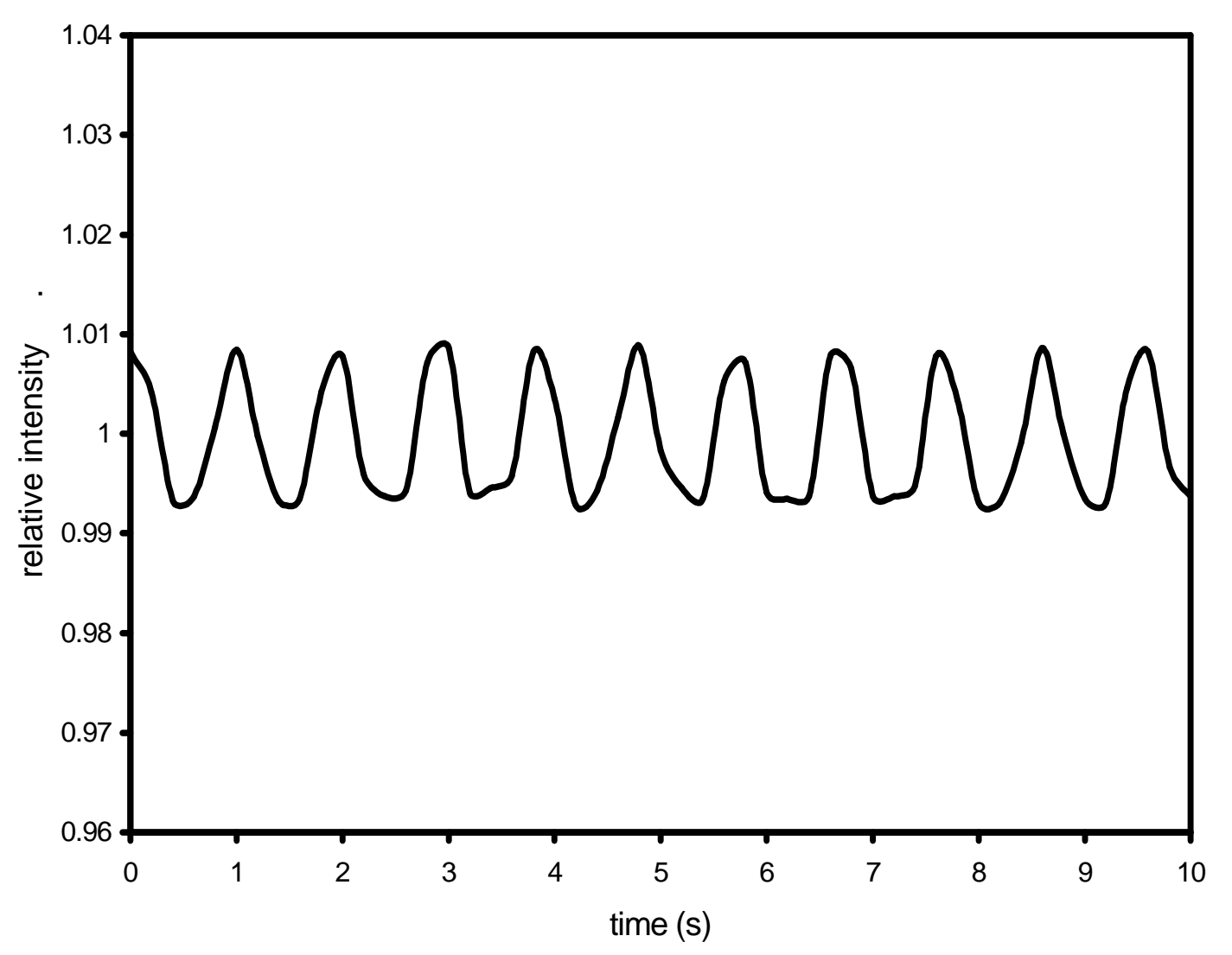




\section{FIGURE CAPTIONS}

Fig. 1 - Simplified functional block diagram of the portable unit. The power supplies, the interface logic between microprocessor and peripherals and the debug / diagnostics hardware are not indicated.

Fig. 2 - Functional diagram of the input filter / amplifiers. The black dots indicate the probe connections, the white dots represent the configuration signals coming from the microcontroller.

Fig. 3 - Transmitted light intensities, normalized to the respective baseline values, measured during tourniquet-induced ischemia. A) ischemia induction, B) release, C) reactive hyperemia, D) recovery.

Fig. 4 - Transmitted light intensity, normalized to the baseline value, measured at 850 $\mathrm{nm}$ during bicycle exercise, showing modulation synchronous with the pedal cycle. 